\title{
El trabajo doméstico y de cuidado no remunerado en los países andinos
}

\author{
Recibido: 15/09/2021 \\ Aprobado: 25/11/2021
}

\author{
REBECA IVONNE CORNEJO LOBO \\ Universidad de Lima (ULIMA) \\ Ministerio de la Mujer y Poblaciones Vulnerables (MIMP) \\ rcornejo@mimp.gob.pe \\ https://orcid.org/0000-0003-0344-1947
}

\section{RESUMEN}

En el presente artículo, la autora analiza la situación del trabajo doméstico y del trabajo de cuidado no remunerado tomando en consideración los resultados de las encuestas de uso de tiempo llevadas a cabo en los países de la subregión andina y su contribución al PIB. Los estudios sobre el uso del tiempo aportan valiosa información sobre cómo las personas distribuyen su tiempo en el trabajo remunerado como no remunerado, demostrando que existe un reparto desigual entre mujeres y hombres. Las mujeres trabajan más horas que los hombres en Perú, Ecuador, Bolivia y Colombia donde existen datos disponibles, sea en la condición en la que se encuentren. Por otro lado, nos permite constatar la importancia del trabajo doméstico y del trabajo de cuidado no remunerado en la reproducción social.

Palabras clave: uso del tiempo, trabajo doméstico no remunerado, trabajo de cuidado no remunerado, carga total de trabajo, participación económica

\section{Unpaid domestic work and care work in the Andean countries}

\begin{abstract}
In this article, the author analyzes the situation of unpaid domestic work and care work taking into consideration the results of the time use surveys carried out in the countries of the Andean Subregion and their contribution to GDP. Time use studies provide valuable information on how people allocate their time for paid and unpaid work, showing that there is an unequal distribution between women and men. Women work longer hours than men in Peru, Ecuador, Bolivia and Colombia, where data is available, regardless of their condition. On the other hand, it allows us to verify the importance of domestic work and unpaid care work in social reproduction.
\end{abstract}

Keywords: time use, unpaid domestic work, unpaid care work, total workload, economic participation 
H mundo del trabajo se ha visto afectado por diversas transformaciones que van más allá del ámbito laboral. Diversos estudios realizados a nivel de la región de América Latina y del Caribe, desde la década de los ochenta, coincidieron en señalar algunos de los efectos más importantes que afectaron el mundo del trabajo como la expansión acelerada del sector terciario, la informalización del empleo con el consiguiente debilitamiento del poder de negociación sindical, la creación de empleo desprotegido bajo modalidades de contratación temporal o a tiempo parcial, y un deterioro de los salarios reales. La flexibilidad salarial debilitó la protección social de los y las trabajadoras de toda la región.

Paralelamente a este proceso, se produjo un incremento sostenido de la tasa de participación y ocupación femenina que se vio favorecida por una serie de factores socioculturales como la expansión de la educación básica, la reducción de las tasas de fertilidad, los cambios experimentados en las percepciones acerca de los roles tradicionales y las mayores aspiraciones de las mujeres en su desarrollo personal y profesional.

A pesar de ello, las mayores tasas de actividad de las mujeres en el mercado laboral no parecen haber cuestionado, de manera sustancial, el modelo del hombre como principal proveedor de los recursos necesarios para el mantenimiento de la familia. Todavía sigue predominando una concepción cultural en la que parte de la identidad femenina está ligada al espacio reproductivo. Estas decisiones que se toman sobre el reparto del tiempo, de los recursos y las aspiraciones sociales van a depender de lo que cada sociedad valora en un determinado momento, de acuerdo con pautas culturales predominantes. De alguna manera dependen de la idea que cada sociedad se crea en torno a la feminidad y la masculinidad ideales.

Desde un punto de vista tradicional, la flexibilización de la jornada laboral ha permitido que las mujeres puedan conjugar las obligaciones domésticas y al mismo tiempo generar ingresos económicos para sus familias sin alterar de manera significativa la forma de organizar el trabajo doméstico y el trabajo de cuidado.

En la actualidad, las mujeres representan alrededor del $50 \%$ de la fuerza laboral de la subregión andina y más del $60 \%$ de la población considerada económicamente inactiva (PEI). Una diferencia marcada está no solo en la proporción de mujeres que conforman la PEI, que es cuantitativamente superior a la de los hombres, sino en su sobrerrepresentación en los llamados quehaceres u oficios del hogar. 
Los estudios sobre el uso del tiempo demuestran que las mujeres trabajan más horas que los hombres en Perú, Ecuador, Bolivia y Colombia. Los estudios realizados en aquellos países de los cuales se cuentan con datos disponibles permiten constatar la importancia del trabajo doméstico y el trabajo de cuidado no remunerado en la reproducción social. El valor económico del trabajo doméstico no remunerado de los hogares, respecto del PIB, oscila entre $19.1 \%$ y $20.4 \%$; de este porcentaje, la mayor contribución corresponde a las mujeres, que fluctúan de $14.1 \%$ a $14.5 \%$, mientras que en el caso de los hombres oscila entre $4.6 \%$ a $6.3 \%$.

La sociedad se beneficia al poner en manos de las mujeres los costos asociados a la reproducción, manteniendo sus salarios por debajo de los de sus pares y confinándolas a ciertos sectores menos valorizados.

\section{Marco teórico}

Las mayores tasas de actividad de las mujeres en el mercado laboral no parecen haber cuestionado el modelo del hombre como principal proveedor de los recursos necesarios para el mantenimiento de la familia. La persistencia de la segregación ocupacional por género deja un margen estrecho de categorías ocupacionales donde las mujeres pueden insertarse. Y esto gracias a la flexibilidad de los horarios, la demanda permanente de mayores niveles de capacitación, la cualidad para soportar la presión y la habilidad para hacer de todo y adaptarse a lo que venga. Sumado a ello, vale decir que los salarios marcadamente bajos para las mujeres se confabulan con los ideales y las expectativas acerca de su condición e identidad en relación con el empleo, el trabajo asalariado y el trabajo de reproducción. No se pueden dejar de lado ciertas voces conservadoras acerca de los «peligros» que está llevando la mayor independencia económica de las mujeres sobre la integración familiar. Algunas de estas voces han puesto el acento en el aumento de la violencia juvenil en las calles, los embarazos tempranos, la autonomía sexual, la violencia contra las mujeres debido a este alejamiento del hogar o de su menor dedicación.

Todavía sigue predominando una opción cultural donde parte de la identidad femenina está ligada al espacio reproductivo. Las decisiones que se toman sobre el reparto del tiempo, de los recursos y las aspiraciones sociales dependen de lo que cada sociedad valora en un determinado momento de acuerdo con pautas culturales predominantes. De alguna manera, dependen 
de la idea que cada sociedad se crea en torno a la feminidad y masculinidad ideales.

Una investigación realizada a finales de los setenta por Jelin y Feijoó (1980), en el área metropolitana de Buenos Aires, recogió información acerca de los cambios en la organización doméstica, el rol de la mujer y las estrategias familiares a través de las historias de vida de mujeres amas de casa y madres de clase obrera de zonas urbanas de escasos recursos. Las autoras utilizan la noción de trabajo que incluye tanto el trabajo extradoméstico, ligado a actividades del mercado, como el doméstico, que involucra tareas vinculadas al mantenimiento y reproducción de los miembros de la unidad doméstica. Según lo señalan las autoras (Jelin y Feijoó 1980, 107) esto «permite detectar los mecanismos por los cuales ambos tipos de tareas se combinan en una misma unidad doméstica y en una misma persona a lo largo de su vida». Los resultados evidenciaron que no se habían producido cambios significativos en las percepciones e imágenes del rol de la mujer. Lo «natural» para una mujer obrera es luchar, sufrir, acomodar las necesidades de los demás miembros de su hogar, subordinar sus deseos privados y su estrategia para el mantenimiento de su familia al ingreso y otras condiciones que pone el marido y, a través de él, el contexto en el que viven. Para las mujeres entrevistadas, el matrimonio no representó un cambio cualitativo en las tareas domésticas: ya de jóvenes y solteras tenían esas responsabilidades en sus familias de origen o se habían desempeñado como empleadas domésticas. Sin embargo, entre las hijas adolescentes había un cuestionamiento sobre por qué los hombres no colaboraban en estas labores, y percibían que era injusta esta división sexual del trabajo.

Un trabajo realizado por Guzmán y Mauro (2004) en Chile analizaron los cambios experimentados en las trayectorias laborales de tres cohortes sucesivas de mujeres y los factores económicos, sociales y políticos que dieron como resultado distintos comportamientos laborales. Estas transformaciones también habrían implicado cambios en las representaciones en torno al rol predominante de hombres y mujeres en la sociedad y los significados que tiene para ambos el trabajo extradoméstico. No todas las jóvenes estarían en mejores condiciones que las generaciones más viejas. Se señalan algunos factores como la menor escolaridad, el nivel socioeconómico, la posición subordinada de la madre dentro de la familia y el punto de inicio del itinerario laboral en empleos precarios, los que habrían contribuido a disminuir sus posibilidades de acceder a posiciones laborales de mayor jerarquía. Las autoras 
advierten que el incremento creciente de la participación de las mujeres en el mercado laboral si bien ha permitido una mayor individuación de las mujeres, no ha logrado cambiar de manera significativa la valoración social del trabajo femenino. La división sexual del trabajo sigue sustentando la diferenciación entre esfera productiva y reproductiva, y la distinta valoración social de los trabajos desempeñados por hombres y mujeres explica la subordinación de los trabajos reproductivos a los realizados en la esfera productiva.

La estrategia de la división sexual del trabajo no ha impedido que las mujeres trabajen intensamente en los talleres familiares, por cuenta propia o en el trabajo doméstico remunerado, aunque estas actividades no han dejado de verse como complementarias al del jefe de familia. Y, además, han formado parte de sus propias estrategias para agenciarse de algunos recursos monetarios, aunque fuesen mínimos y de alcanzar cierta cuota de independencia frente a otros miembros de la familia.

La doble presencia de la mujer para sostener el equilibrio a lo largo de su vida entre el trabajo doméstico no remunerado orientado a la reproducción de la familia y el trabajo remunerado es algo que puede verse en las tasas de actividad por rangos de edades para cada uno de los países referidos. Su entrada y/o salida del mercado de trabajo se ha ido moldeando de acuerdo con las exigencias del ciclo reproductivo y, a su vez, por los cambios experimentados en la actividad económica. Así, para Picchio (1994, 463):

la persistencia en el tiempo y el carácter generalizado de su distribución desigual entre hombres y mujeres revelan su papel básico en la estructura de género del mercado laboral, toda vez que la condición de los hombres demuestra ser casi totalmente impermeable a las condiciones de la familia, mientras esta constituye el elemento fundamental para la ubicación de las mujeres en el mercado laboral.

El aporte desde la teoría feminista a los estudios acerca del trabajo femenino planteó una fuerte crítica a los paradigmas de la teoría neoclásica y la teoría marxista sobre la interpretación que se hicieron sobre la situación económica de las mujeres. El debate ha sido amplio y las críticas formuladas hicieron hincapié al tratamiento que se hizo sobre el sistema familiar, al concepto del trabajo restringido solo al ámbito de la producción mercantil y a la supuesta neutralidad del sistema económico que crea y mantiene trabajos mal remunerados debido a la existencia de determinados grupos sociales que solo ten- 
drían un interés marginal y se contentarían con trabajos inestables y precarios. En ese sentido, Benería (1987) afirma lo siguiente:

Cualquier actividad realizada fuera de la esfera mercantil no es considerada económica para quienes participan en ella- básicamente mujeres- no se definen como trabajadoras(os) activas(os). Es decir, solo se incluye como actividad económica aquella destinada a producir mercancías, bienes con valor de cambio y se ignora los procesos que producen bienes solo con valor de uso. Lo cual, traducido a términos más genéricos, implica que toda la actividad económica tiene como centro la esfera mercantil, siendo la esfera de reproducción subsidiaria y dependiente de ésta. Cualquier conceptualización de actividad económica debe incluir todos los procesos de producción de bienes y servicios orientados a la subsistencia y reproducción de las personas, independientemente de las relaciones bajo las cuales se produzcan (en Borderías et al. 1994, 79).

La familia no se concibe tan solo como una unidad de consumo que se organiza para maximizar la utilidad familiar. A su vez, la utilidad familiar no es solo la agregación de preferencias colectivas que toman en cuenta las preferencias individuales y establece un orden equitativo. Las contradicciones y conflictos suelen afectar la posición de los miembros de la unidad doméstica tanto en el mercado laboral como al interior de la familia. Las decisiones que se toman en el ámbito familiar aún se basan en un sistema desigualdades entre los sexos, pero al mismo tiempo entran en contradicciones por los requerimientos del propio mercado de trabajo. En este contexto de flexibilización laboral, el valor productivo de los varones ha disminuido relativamente y se han puesto mayores expectativas de mejora económica para las mujeres, pero la intensidad y el tipo de actividad productiva que realizan muestra el complicado equilibrio entre producción y reproducción.

\section{Metodología}

La presente investigación es de tipo descriptiva, basada en el análisis de información secundaria de fuentes oficiales como los resultados de las encuestas de uso del tiempo de Perú, Colombia, Ecuador y Bolivia, y de información complementaria sobre tasas de participación laboral de la DANE (Departamento Administrativo Nacional de Estadística) de Colombia, INE (Instituto Nacional 
de Estadísticas) de Bolivia, INEC (Instituto Nacional de Estadística y Censo) de Ecuador, INEI (Instituto Nacional de Estadística e Informática) del Perú, así como del portal estadístico CEPALSTAT de la CEPAL y de ILOSTAT de la OIT. Por otro lado, se ha utilizado una serie histórica de las tasas de actividad económica de mujeres y hombres de 1990-2019 para un rango de la población comprendida entre los 15 a 64 años de edad, la cual permitirá la comparabilidad entre los países de la subregión andina y cuya fuente proviene de ILOSTAT de la OIT.

\section{Resultados y discusión}

Este apartado se divide en tres secciones: i) análisis de las tasas de participación de mujeres y hombres; ii) análisis de los resultados de las encuestas de uso del tiempo de Colombia, Ecuador y Perú; y iii) el valor económico del trabajo doméstico no remunerado.

\subsection{Tasa de participación de hombres y mujeres en la fuerza de trabajo}

Desde mediados de la década de los ochenta, la participación de la mujer en el mercado laboral se ha incrementado en forma sostenida, cambiando incluso el patrón de incorporación de la población activa de la clásica curva en forma de $\mathrm{M}$ a una en forma de $\mathrm{U}$ invertida, semejándose cada vez más a la de los varones en muchos países del mundo. Esto quiere decir que las mujeres cada vez más permanecen activas por más tiempo y sin interrupciones.

Según cifras de la OIT (2019), en América Latina y el Caribe, la participación femenina en la fuerza laboral pasó de un $50.7 \%$ en el 2000 a un $57.9 \%$ en el 2019. Para los países de la subregión andina, la participación femenina en la fuerza laboral ha tenido un comportamiento variable, pero creciente en el largo plazo. Así, se han superado los niveles esperados, reduciéndose de manera continua la brecha existente en las tasas de actividad entre hombres y mujeres. Para finales de la década, la presencia de la mujer en la población activa era superior al $50 \%$, con excepción de Venezuela.

En algunos países el aumento fue mayor. Así, en Bolivia, ya para el 2000, habían superado el $60 \%$ alcanzando su nivel más alto en el 2018, con 65.3 $\%$. En el Perú, en el año 2001, la tasa de actividad femenina era de $62.2 \%$, y para el año 2019 había alcanzado su nivel más alto, en $74.1 \%$, aumentando 
en 11.9 \% en comparación al año 2001 (INEI 2020). En Venezuela, la participación de las mujeres aumentó de $47.1 \%$ en 1990 a $53.3 \%$ en el 2013 , pero ha descendido en los años 2018 y 2019 a $49.6 \%$ y $46.4 \%$, respectivamente. Este incremento sostenido en la mayoría de los países andinos de las tasas de participación femenina ha traído como consecuencia que la brecha de la participación laboral entre mujeres y hombres disminuya.

FIGURA 1

Subregión andina: tasas de actividad económica de la población de 15 a 64 años, por sexo y brechas
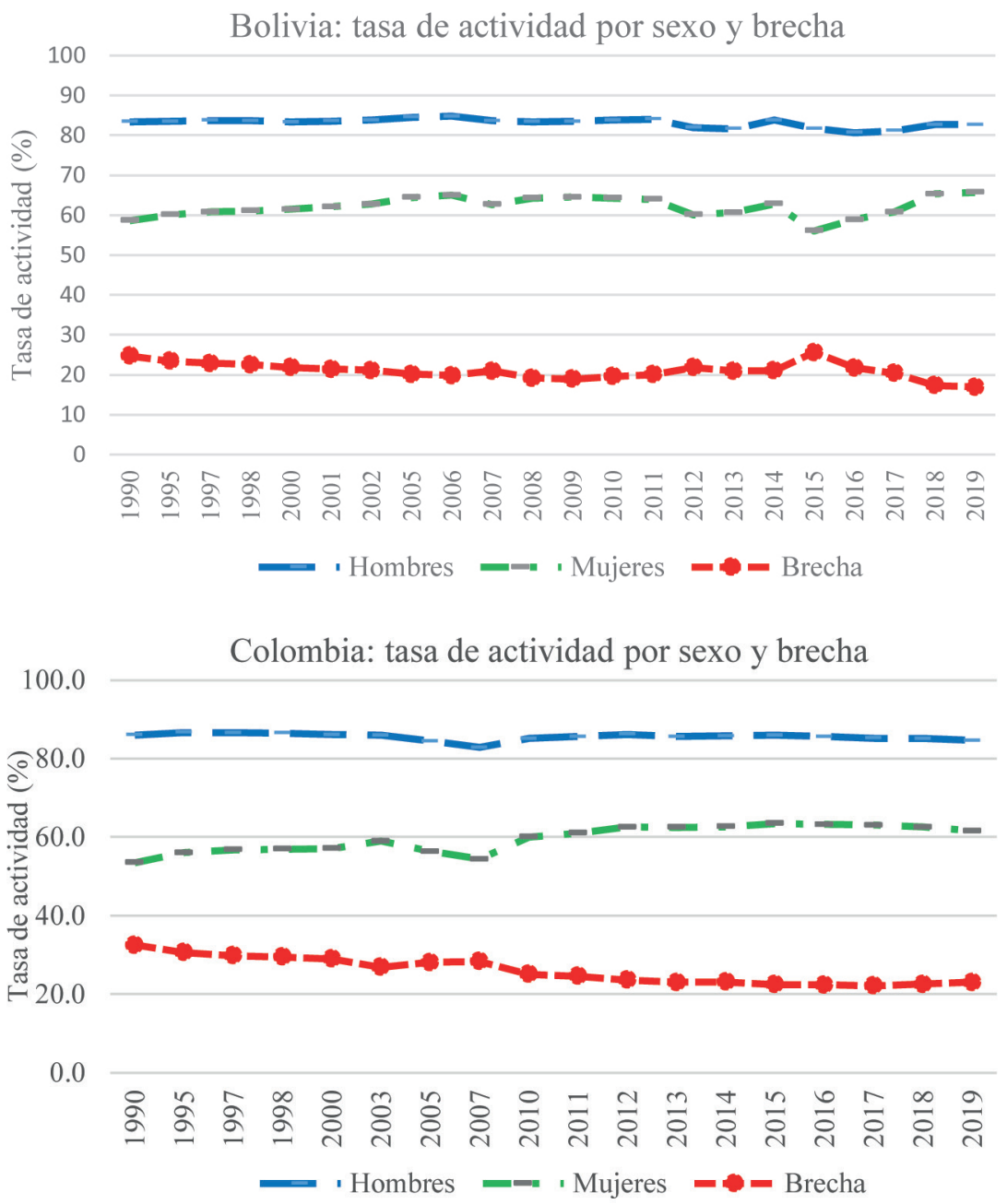
100.0

Ecuador: tasa de actividad por sexo y brecha

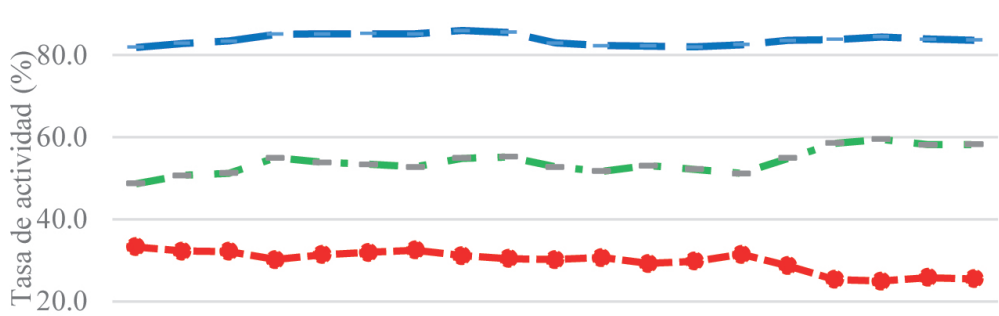

0.0

ஓ

- Hombres - ' Mujeres

100.0 Perú: tasa de actividad por sexo y brecha

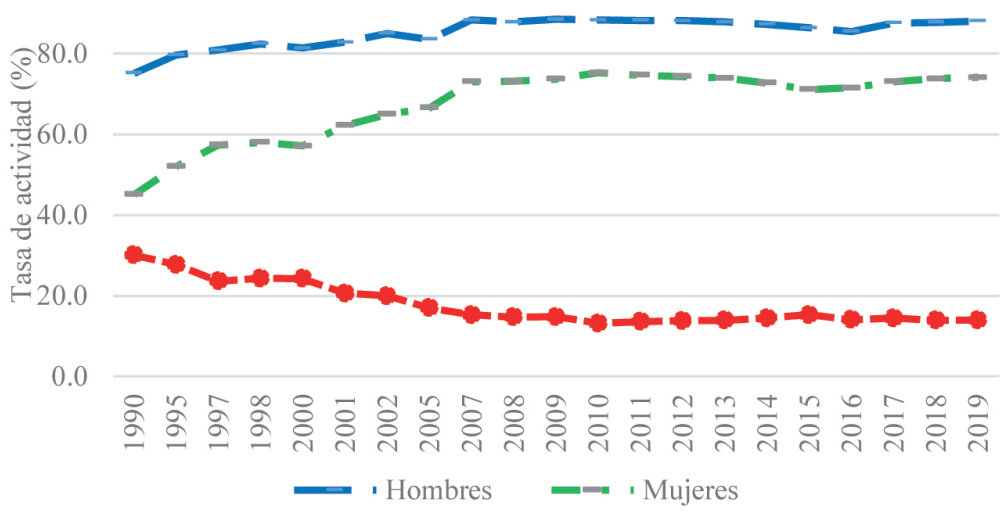

100

Venezuela: tasa de actividad por sexo y brecha

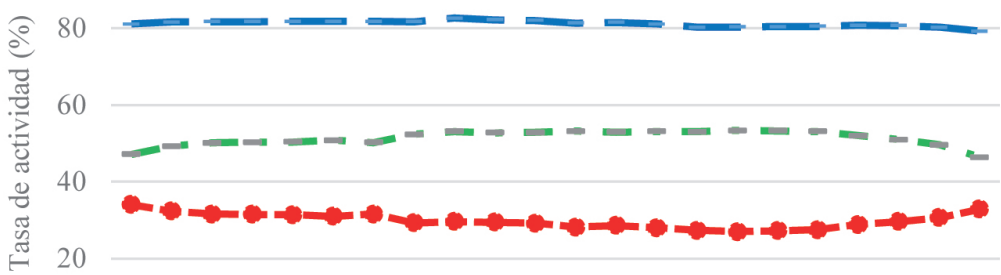

0

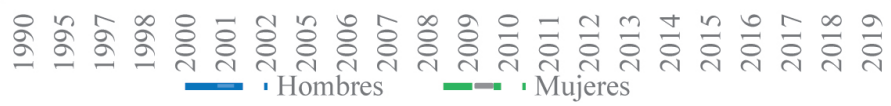

Fuente: ILOSTAT (2021) 
Son varios los factores que han contribuido a este incremento, pero entre los más importantes que se suelen mencionar están la reducción en los niveles de fertilidad, ${ }^{1}$ el incremento en el nivel educativo de las mujeres, la caída en los ingresos reales de las familias y la pérdida de empleo formal, sobre todo, de los hombres. Hay una permanencia mayor de las mujeres en sus trayectorias laborales durante la época de mayor actividad reproductiva, tendencia por un lado a tener menos hijos, a retrasar la edad de los nacimientos, como también la de asumir simultáneamente la vida laboral y las responsabilidades domésticas.

Una estimación realizada por la CEPAL $(2014,203)$, reveló que:

una variación en las tasas de participación femenina en edades centrales (entre 14 y 65 años) que se equipare a la de los hombres, produciría un incremento del ingreso medio de los hogares que va desde un porcentaje entre el $3 \%$ y el $4 \%$ en la Argentina, el Brasil, Colombia, México y el Uruguay, hasta niveles superiores al $10 \%$ en El Salvador, Honduras, Nicaragua y el Perú.

Para el caso de los países de la subregión andina, se producirían descensos más pronunciados en la incidencia de la pobreza de Ecuador y Perú (de 9 a 6 $\%$ ), y en 5.4 y $4 \%$ en Bolivia, Colombia y Venezuela, respectivamente.

Uno de los factores señalado anteriormente tiene que ver con el nivel educativo, que favorecía al incremento de las tasas de participación laboral de las mujeres, como es el caso de Bolivia y Venezuela. El incremento es notable cuando se alcanza el nivel de bachiller, aun cuando no logran a alcanzar al nivel observado en los hombres. Para el caso de Bolivia, la tasa de participación laboral femenina se incrementa de manera notable a $76 \%$; con respecto al nivel educativo de educación secundaria, el incremento es de $19.2 \%$ en mujeres, mientras que los hombres alcanzan el nivel de $88,9 \%$; pero el cambio de un nivel educativo a otro no es tan notable (8.7\%). En Venezuela, se observa algo similar: la tasa de participación laboral femenina alcanza el nivel de 68.8 $\%$ cuando el nivel educativo se eleva, reduciéndose de manera significativa

1 En el Perú, la tasa global de fecundidad (TGF) en 1990 era de 3.9 hijos por mujer, en el 2010 descendió a 2.5 hijos promedio por mujer y la estimación para el 2020 es de 2.2 hijos; en Ecuador, en 1990 era de 3.7 hijos y en el 2010 era de 2.6 hijos y para el 2020 se estima en 2.4 hijos; en Colombia, en 1990 era 3.08 hijos, en el 2010 era de 1.99 hijos y para el 2019 esta se redujo a 1.79 hijos; en Venezuela, en 1990 era de 3.45 hijos y en el 2010 de 2.47 hijos y en el 2020 se redujo a 2.23 hijos; en Bolivia, en 1990 era de 4.9 hijos, en el 2010 era de 3.2 hijos y en el 2020 se redujo a 2.7 hijos. 
la brecha de género con respecto a la observada en los hombres de $28 \%$ con secundaria completa a $9.1 \%$ con bachillerato.

\section{FIGURA 2}

Bolivia y Venezuela: tasa de participación laboral de las mujeres de 15 años y más por nivel educativo, 2019 y 2017

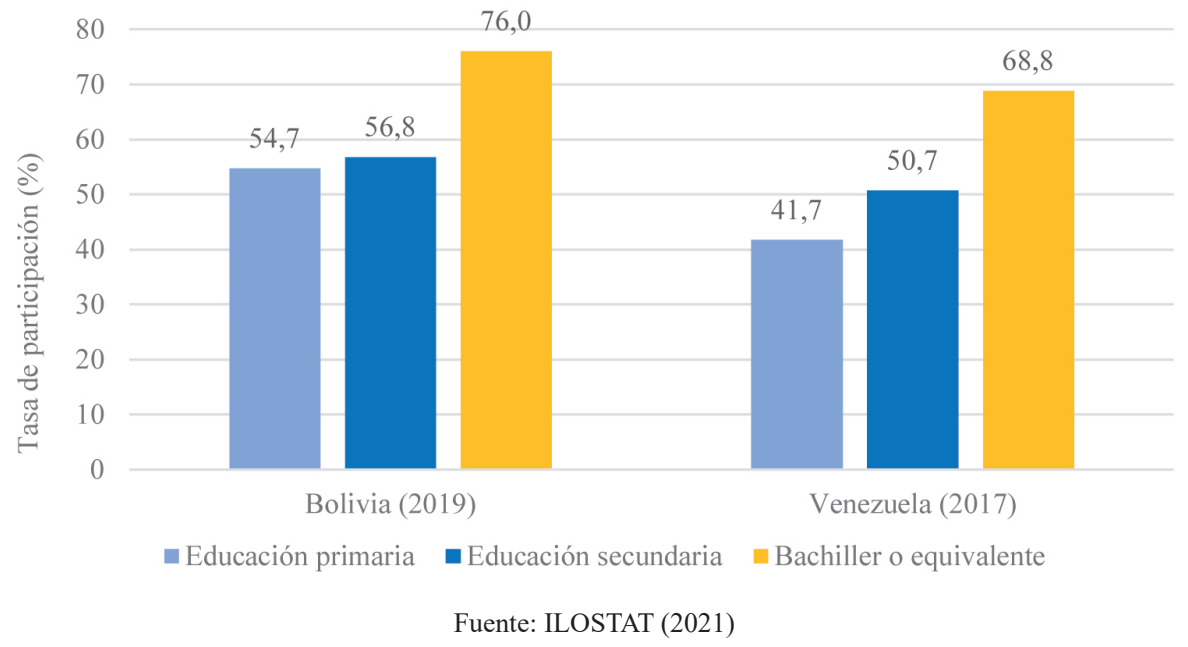

\subsection{Los datos empíricos: resultados del módulo uso del tiempo}

En diversos países de la región de América Latina y el Caribe, se han venido impulsando iniciativas para cuantificar el tiempo asignado al trabajo reproductivo con la finalidad de lograr su reconocimiento y cuantificación. Además, estos esfuerzos también se han dado en el desarrollo conceptual y metodológico para la medición del uso del tiempo, su armonización y normalización, así como la construcción de una cuenta satélite del trabajo doméstico no remunerado.

En la IV Conferencia Mundial sobre la Mujer de la ONU (Beijing, 1995) y en la Cumbre Mundial sobre Desarrollo Social (Copenhague, 1995), se solicitó a los Gobiernos la elaboración de indicadores y cuentas satélite capaces de reflejar en los sistemas de contabilidad nacional la contribución cuantitativa derivada del trabajo no remunerado, particularmente del trabajo doméstico, como ocurre con el trabajo productivo.

Es a partir de la década de 1980 que en América Latina y el Caribe se comienzan a realizar encuestas de uso del tiempo. Así, Cuba (1985) fue el 
primer país de la región que emprendió la medición del uso del tiempo a través de la Oficina Nacional de Estadística (ONE). Posteriormente, se sumaron otros países como México (1996) y Nicaragua (1998), quienes fueron pioneros en este tipo de encuestas.

El Comité Ejecutivo del CEA (Conferencia Estadística de las Américas, 2008), de la CEPAL, en su octava reunión celebrada en octubre del 2008, aprobó el Programa de Trabajo del Grupo de Trabajo sobre Estadísticas de Género. En la quinta reunión de la CEA realizada en agosto del 2009, se propusieron desarrollar capacidades técnicas y metodológicas para producir estadísticas sobre el uso del tiempo, el trabajo no remunerado, la violencia contra las mujeres, así como diagnósticos sobre la situación de las estadísticas e indicadores de género en los países de la región. Además, hacer seguimiento sobre el impacto de la crisis económica en el empleo y el desempleo femenino, y la cobertura de los programas sociales y de empleabilidad.

En el informe y las conclusiones de la Comisión de la Igualdad de Género de la OIT realizados en junio del 2009, se reconocen que los esfuerzos encaminados a lograr «la igualdad de género son parte esencial del trabajo decente». Se espera que los países miembros avancen en la igualdad salarial, creen condiciones para la participación activa de los hombres en las responsabilidades domésticas y en la crianza de los y las hijas, establezcan políticas que desincentiven la discriminación y los estereotipos basados en el sexo por parte de los empleadores y en la educación básica, técnica y profesional. Las estrategias desplegadas deberán tomar en cuenta la perspectiva del ciclo de vida de hombres y mujeres, prestando especial atención a las situaciones de transición en la vida de ambos. Así mismo, se esperan cambios en la legislación que apuesten por conciliar las responsabilidades laborales y familiares de hombres y mujeres. Por último, los Gobiernos deberán intensificar los esfuerzos para ofrecer y garantizar servicios de calidad para el cuidado de niños y niñas, y de otras personas a cargo.

Posteriormente, en la Octava Reunión del CEA (Conferencia Estadística de las Américas) de la CEPAL, celebrada en Quito en el 2015, todos los países miembros adoptaron la CAUTAL (Clasificación de Actividades de Uso del Tiempo para América Latina) con enfoque de género y adecuada al contexto regional. Esto ha significado un avance importante en la homologación de las bases de datos del repositorio de información de la CEPAL (CEPAL 2019, 3).

La definición de la población económicamente activa adoptada por la Décimo Tercera Conferencia Internacional de Estadígrafos del Trabajo-CIET/ 
OIT (ILO 1982, 2), aquella que los países andinos han ido adecuándose en el tiempo, estableció lo siguiente:

La población económicamente activa abarca todas las personas de ambos sexos que aportan su trabajo para producir bienes y servicios económicos de acuerdo con los sistemas de cuentas nacionales durante un período de referencia especificado. Por bienes y servicios económicos se entiende a toda la producción y tratamiento de productos primarios que pueden destinarse al mercado, al trueque o al autoconsumo, y en el caso de los hogares que produzcan artículos y servicios para el mercado, la parte de esta producción destinada al autoconsumo.

Este concepto de actividad económica está vinculado a quienes se encuentran con empleo remunerado o quienes están desempleados, pero que están buscando activamente un empleo remunerado. Tal definición deja de lado aquellas actividades por las cuales no se percibe ninguna remuneración y no media ningún vínculo con el empleador.

La distinción entre actividad económica y no económica se base en el uso del tiempo entre el trabajo productivo y el ocio. La economía habría tomado en cuenta solo la experiencia masculina, en la que las decisiones de entrada y/o salida del mercado de trabajo dependen del costo de oportunidad entre los ingresos del trabajo remunerado y el ocio, mientras que a las mujeres se les plantea el tener que decidir entre el trabajo asalariado, las presiones por el trabajo doméstico no remunerado, así como el cuidado de la familia y el ocio, que es casi una opción marginal.

En la actualidad, las mujeres representan alrededor del $50 \%$ de la fuerza laboral de la subregión andina y más del $60 \%$ de la población considerada económicamente inactiva (PEI). Aunque no hay una sola manera de conceptualizar a la población económicamente inactiva entre los países miembros de la CAN, según las normas internacionales convienen en señalar que está conformada por estudiantes, personas dedicadas a los quehaceres del hogar, pensionistas, rentistas, personas con algún problema de salud, personas de muy avanzada edad o personas en situación de discapacidad permanente para trabajar. No todos los países presentan su información desagregada, de tal forma que se puede conocer las razones de inactividad de hombres y mujeres o la información ha sido discontinuada, por lo que no es posible observar cambios en su participación. Sin embargo, lo que es común a los países que sí cuentan con información más detallada es que los motivos de inactividad difieren en- 
tre hombres y mujeres. Así, mientras que para los primeros la causa principal está vinculada a los estudios, para las mujeres la dedicación a los quehaceres del hogar es el motivo principal. Las cifras muestran que el trabajo doméstico no remunerado es casi una exclusividad de las mujeres.

En Colombia, se sabe que alrededor del $60 \%$ de las mujeres que se encontraban inactivas estaban dedicadas a los oficios del hogar, a diferencia de los hombres que en un $54 \%$ se encontraban estudiando (DANE, 2019-2020). De manera similar en el Perú, el 59.4\% de la PEI femenina estaba dedicada a los quehaceres y $26.4 \%$ estaban estudiando. En el caso de los hombres, un $17.5 \%$ se dedicaban a los quehaceres del hogar y un $51.6 \%$ estudiaban (INEI 2017, 41).

TABLA 1

Subregión andina (4 países): población en edad de trabajar (PET) y porcentaje de la población económicamente inactiva femenina

\begin{tabular}{|c|c|c|c|c|c|}
\hline \multirow{2}{*}{ Países } & \multicolumn{3}{|c|}{ PET (en miles de personas) } & \multicolumn{2}{|c|}{$\begin{array}{c}\text { PEI (en miles de } \\
\text { personas) }\end{array}$} \\
\hline & Total & Hombres & Mujeres & Total & $\begin{array}{l}\text { Mujeres } \\
\text { (\% PEI) }\end{array}$ \\
\hline Perú $(2017)^{\mathrm{a}}$ & 23772 & 11832 & 11939 & 6556 & 65.6 \\
\hline Ecuador (dic 2019) ${ }^{b}$ & 12403 & 6066 & 6336 & 4304 & 69.0 \\
\hline $\begin{array}{l}\text { Colombia (dic 2019- } \\
\text { feb 2020) }^{\mathrm{c}}\end{array}$ & 39626 & 19381 & 20245 & 14636 & 66.1 \\
\hline Bolivia $(2017)^{d}$ & 8958 & 4352 & 4607 & 3371 & 64.0 \\
\hline
\end{tabular}

a INEI (2017). La PET comprende a la población de 14 años y más.

${ }^{\mathrm{b}}$ INEC (2019). La PET comprende a todas las personas de 15 años y más.

c DANE (2020). La PET está constituida por las personas de 12 años y más en las zonas urbanas y 10 años y más en las zonas rurales.

${ }^{\mathrm{d}}$ INE (2017). Comprende a la población mayor de 10 años, habilitada física e intelectualmente para ejercer una actividad económica.

La proporción de mujeres en edad de trabajar que no tienen un trabajo remunerado es considerablemente superior a la de los hombres, y para una mayoría de los países andinos representa casi la tercera parte de la PET femenina (incluye quehaceres del hogar y trabajo familiar no remunerado). Gran parte se debe al trabajo doméstico no remunerado que realiza la población considerada no activa. Estos porcentajes están subvaluados al no tomar en cuenta que una proporción de mujeres ocupadas y desempleadas no dejan de hacerse cargo de estas labores, pero además porque el trabajo doméstico comprende una parte de las obligaciones y las tareas asociadas a la reproducción. Estos 
porcentajes también revelan el poco involucramiento de los hombres en estas labores.

La figura 3 muestra las diferencias entre hombres y mujeres en la distribución del tiempo entre los trabajos remunerado y no remunerado de tres países de la subregión andina. En Ecuador, Colombia y Perú, las mujeres ocupan la mayor parte de su tiempo al trabajo no remunerado, en alrededor del $60 \%$, mientras que los hombres le dedican menos de la cuarta parte de sus horas semanales.

En el Perú, se aprecia que el tiempo promedio que le dedican las mujeres al trabajo no remunerado es de más de 22 horas, en relación con el tiempo que le dedican los hombres, quienes destinan más de 44 horas al trabajo remunerado. Sin duda, la diferencia entre ellos es de una brecha muy amplia, lo mismo que se puede ejemplificar en los demás países de la subregión andina.

Figura 3

Subregión andina (3 países): tiempo total de trabajo según tipo de trabajo y sexo (en horas semanales)

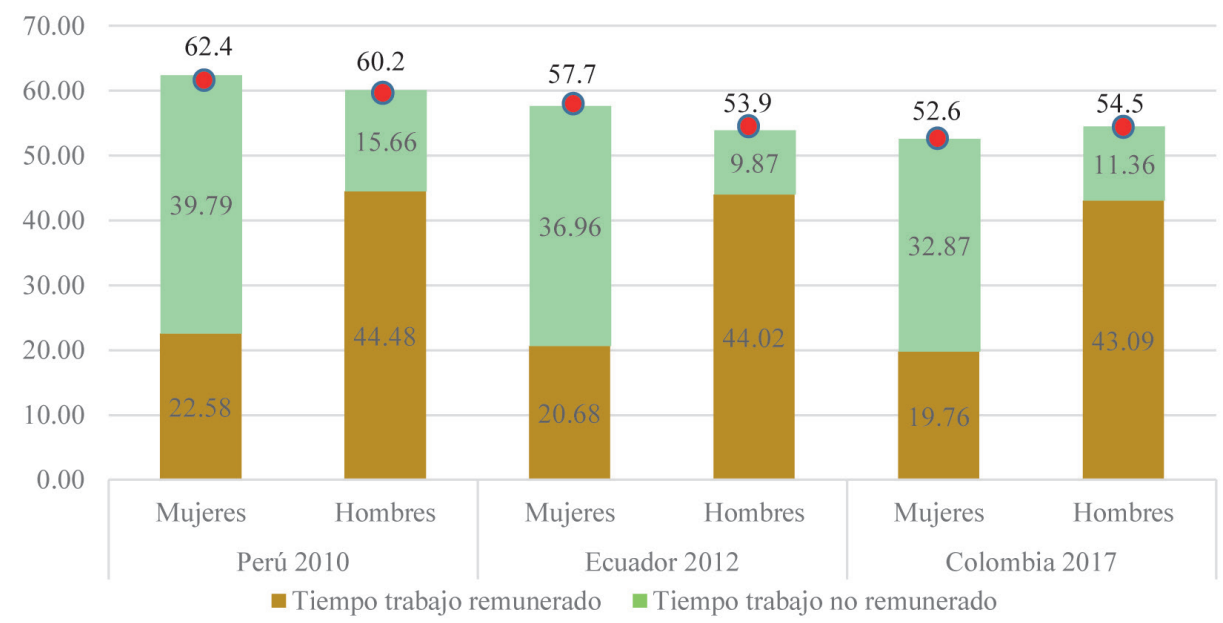

Fuente: CEPALSTAT (2019). https://cutt.ly/dUnnFGA

En Colombia se aprecia que, en los hogares biparentales con o sin hijos/ as, los hombres reducen el tiempo destinado al trabajo no remunerado en casi cinco horas semanales que cuando estaban solos o no habían formado una familia. En el caso de las mujeres, el tiempo dedicado al trabajo no remunerado se incrementa en más de trece horas, sobre todo cuando tienen hijos/as. Lo 
más significativo es que el trabajo no remunerado de los hombres se reduce aún más cuando aumenta el número de miembros de la familia, ya sea por la presencia de parientes u otros sin un vínculo de parentesco. Es probable que ese tiempo que se ahorran recaiga sobre otras mujeres distintas a la cónyuge o la(s) hija(s). Las mujeres también reducen sus horas diarias al repartir las labores entre otros miembros que comparten el hogar; pero en relación con los hombres que se encuentran en la misma situación, ellas trabajarían casi el triple de horas semanales.

De acuerdo con la Encuesta Nacional de Uso del Tiempo realizada en Ecuador en el 2012, se aprecia de manera similar a los resultados de Colombia que los hombres reducen de manera significativa el tiempo que le destinan al trabajo no remunerado en los hogares biparentales con hijos/as en casi trece horas a la semana. De igual manera, en hogares compuestos por encima de esta cifra, pueden destinar más horas al trabajo remunerado.

Por otro lado, en el Perú el trabajo no remunerado que realizan los hombres en hogares biparentales sin hijos/as es menos de la tercera parte del que realizan las mujeres. Y en hogares con hijos/os, la carga de trabajo para las mujeres (39.05 horas semanales) se eleva más de cuatro veces en relación con los hombres ( 8.88 horas semanales) en el mismo tipo de hogar.

TABLA 2

Subregión andina: tiempo total de trabajo según tipo de hogar* y sexo (en horas semanales)

\begin{tabular}{|r|r|r|r|r|r|r|}
\hline \multirow{2}{*}{ País } & \multicolumn{2}{|c|}{$\begin{array}{c}\text { Tiempo total de } \\
\text { trabajo }\end{array}$} & \multicolumn{2}{c|}{$\begin{array}{c}\text { Tiempo de trabajo } \\
\text { remunerado }\end{array}$} & \multicolumn{2}{c|}{$\begin{array}{c}\text { Tiempo trabajo no } \\
\text { remunerado }\end{array}$} \\
\cline { 2 - 7 } & Hombre & \multicolumn{1}{|c|}{ Mujer } & Hombre & \multicolumn{1}{c|}{ Mujer } & Hombre & Mujer \\
\hline Colombia 2017 & \multicolumn{5}{|c|}{} & \multicolumn{2}{|c|}{} \\
\hline Unipersonal & 58.00 & 46.76 & 41.74 & 22.94 & 16.26 & 23.81 \\
\hline $\begin{array}{r}\text { Biparental sin } \\
\text { hijos/as }\end{array}$ & 53.98 & 49.95 & 42.63 & 19.20 & 11.35 & 30.75 \\
\hline $\begin{array}{r}\text { Biparental con } \\
\text { hijos/as }\end{array}$ & 57.08 & 55.54 & 45.77 & 18.25 & 11.31 & 37.29 \\
\hline $\begin{array}{r}\text { Extendido } \\
\text { Compuesto }\end{array}$ & 50.93 & 50.19 & 40.63 & 19.63 & 10.29 & 30.56 \\
\hline $\begin{array}{r}\text { Monoparental } \\
\text { con hijos/as } \\
\text { menores de 15 } \\
\text { años }\end{array}$ & 44.26 & 50.22 & 46.92 & 18.80 & 10.34 & 31.42 \\
\hline Sin vínculo & 57.09 & 49.66 & 45.12 & 27.06 & 11.97 & 22.59 \\
\hline
\end{tabular}




\begin{tabular}{|c|c|c|c|c|c|c|}
\hline \multicolumn{7}{|l|}{ Ecuador 2012} \\
\hline Unipersonal & 63.89 & 53.38 & 42.41 & 23.18 & 21.48 & 30.20 \\
\hline $\begin{array}{r}\text { Biparental sin } \\
\text { hijos/as }\end{array}$ & 51.47 & 55.00 & 40.02 & 17.99 & 11.45 & 37.01 \\
\hline $\begin{array}{r}\text { Biparental con } \\
\text { hijos/as }\end{array}$ & 55.06 & 57.78 & 46.18 & 18.73 & 8.88 & 39.05 \\
\hline Extendido & 47.29 & 55.51 & 38.08 & 24.68 & 9.22 & 30.83 \\
\hline Compuesto & 56.97 & 55.62 & 49.05 & 21.66 & 7.92 & 33.97 \\
\hline $\begin{array}{r}\text { Monoparental } \\
\text { con hijos/as me- } \\
\text { nores de } 15 \text { año }\end{array}$ & 46.61 & 67.31 & 33.99 & 29.38 & 12.62 & 37.93 \\
\hline Sin vínculo & 53.67 & 47.03 & 43.31 & 20.31 & 10.36 & 26.72 \\
\hline \multicolumn{7}{|l|}{ Perú 2010} \\
\hline Unipersonal & 71.31 & 60.71 & 49.55 & 24.53 & 21.76 & 36.19 \\
\hline $\begin{array}{r}\text { Biparental sin } \\
\text { hijos/as }\end{array}$ & 62.36 & 63.68 & 44.34 & 22.86 & 18.02 & 40.82 \\
\hline $\begin{array}{r}\text { Biparental con } \\
\text { hijos/as }\end{array}$ & 61.57 & 64.12 & 46.06 & 20.44 & 15.51 & 43.68 \\
\hline Extendido & 57.13 & 60.50 & 42.47 & 23.58 & 14.66 & 36.92 \\
\hline Compuesto & 55.23 & 53.45 & 40.91 & 23.31 & 14.32 & 30.15 \\
\hline $\begin{array}{r}\text { Monoparental } \\
\text { con hijos/as } \\
\text { menores de } 15 \\
\text { años }\end{array}$ & 56.88 & 68.73 & 36.46 & 30.50 & 20.43 & 38.23 \\
\hline Sin vínculo & - & - & - & - & - & - \\
\hline
\end{tabular}

* Las categorías de tipos de hogares están diferenciados según el parentesco de los miembros del hogar. Unipersonales: hogares compuestos por una sola persona; biparental sin hijos: hogares compuestos por jefe/a de hogar y cónyuge; biparental con hijos: hogares compuestos por jefe/a de hogar, cónyuge e hijos; extendido: hogares compuestos por jefe/a de hogar, cónyuge, hijos y otros parientes, en el cual incluye hogares con jefe/a de hogar e hijos mayores de 15 años; compuesto: hogares compuestos por jefe/a de hogar, cónyuge, hijos, otros parientes y otros no parientes; monoparental: jefe/a de hogar e hijos menores de 15 años; sin vínculos: hogares con miembros sin vínculo. Ficha técnica del indicador: CEPALSTAT (2019). https://cutt.ly/vUnmGUx

Nota: Está referido a la población de 15 años y más.

Fuente: CEPALSTAT (2019). https://cutt.ly/dUnnFGA

\subsection{Valorización deltrabajodomésticonoremuneradoysu contribución alPIB}

Entre los países andinos de los cuales se cuentan con datos disponibles, se aprecia que el valor económico del trabajo doméstico no remunerado de los hogares respecto del PIB osciló entre 19.1 \% y 20.4 \%. Así, en Ecuador, de acuerdo con cálculos desarrollados por el Instituto Nacional de Estadística y 
Censos (INEC), en el año 2017, el aporte del trabajo doméstico no remunerado al PIB representó el $19.1 \%$, cifra superior al resto de actividades económicas como de la industria manufacturera, que tuvo una participación de $14.4 \%$, y la de construcción, que fue de $11.6 \%$. De esta manera, han sido las mujeres las que más aportan con el $14.5 \%$, a diferencia de los hombres que aportan el 4.6\%. Son las actividades culinarias las que mayor aportan a la producción con el $58.5 \%$; y son las mujeres las que contribuyen de manera significativa $(86.3 \%)$ con respecto a los hombres $(13.7 \%)$.

\section{Tabla 3}

Valorización económica del trabajo doméstico no remunerado de los hogares realizados por los países andinos, según sexo

\begin{tabular}{|l|c|c|c|c|c|}
\hline \multirow{2}{*}{ País } & \multirow{2}{*}{ Año } & & \multicolumn{3}{c|}{ \% PIB } \\
\cline { 3 - 5 } & & & Total & Mujer & Hombre \\
\hline Ecuador $^{\mathrm{a}}$ & 2017 & & 19,1 & 14,5 & 4,6 \\
\hline Colombia $^{\mathrm{b}}$ & 2017 & 20,0 & & \\
\hline Perú $^{\mathrm{c}}$ & \multicolumn{2}{|c|}{2010} & 20,4 & 14,1 & 6,3 \\
\hline
\end{tabular}

Fuente: CEPAL (2020, 215)

${ }^{\text {a }}$ Los datos provienen de INEC (2019).

${ }^{\mathrm{b}}$ Los datos provienen de la DANE (2017)

${ }^{\mathrm{c}}$ Los datos provienen del INEI (2016).

En el 2016, el Perú a través del INEI elaboró la Cuenta Satélite del Trabajo Doméstico no Remunerado de los Hogares, con la finalidad de valorar el trabajo doméstico no remunerado que realizan mujeres y hombres, así como su aporte al PBI del país. Los resultados muestran que el valor del trabajo doméstico no remunerado de los hogares ${ }^{2}$ representó el $20.4 \%$ del PBI del año 2010, siendo el $14.1 \%$ generado por las mujeres y el $6.3 \%$ por los hombres (INEI 2016).

De acuerdo con el INEI $(2016,45)$, la función que representa un mayor porcentaje del PBI en el trabajo no remunerado es la de proveer comida y refrigerios $(6.5 \%)$, seguido del cuidado de niños/as y personas adultas mayores $(5.1 \%)$. En la función de proveer comida y refrigerios, las horas que destinan las mujeres es de 4.2 veces superior a la de los hombres, y 2.5 veces en proveer cuidados a niños/as y personas adultas mayores.

2 La Cuenta Satélite del trabajo Doméstico no Remunerado de los Hogares define las actividades que realizan los hogares en la función de producción de servicios domésticos, como proveer comidas, proveer vestimenta y cuidado de prendas, proveer cuidado de niños, niñas y personas adultas mayores, con el propósito de compararlas con los servicios similares producidas para el mercado. 
En el caso de Colombia, según el Departamento Administrativo Nacional de Estadística (DANE 2017), la producción de trabajo doméstico y de cuidado no remunerado equivale al $20 \%$ del PBI. Por otro lado, al compararlo con otras actividades que aportaron el mayor valor económico, como el comercio al por mayor y menor, reparación de vehículos, transporte y almacenamiento, alojamiento y servicios de comida, el aporte del trabajo doméstico y de cuidado no remunerado fue superior (185 722 vs. 162507 miles de millones de pesos colombianos).

\section{Reflexiones finales}

Los estudios sobre el uso del tiempo demuestran que las mujeres trabajan más horas que los hombres en Perú, Ecuador, Bolivia y Colombia. Las estadísticas demuestran que incluso la carga del trabajo total es mayor para las mujeres que realizan trabajo extradoméstico o estaban buscando un trabajo remunerado. Para los hombres, cuando se unen en pareja, el tiempo que le dedican al trabajo doméstico se reduce o no hay mucha diferencia que cuando estaban solteros.

Los estudios realizados en aquellos países de los cuales se cuentan con datos disponibles permiten constatar la importancia del trabajo doméstico en la reproducción social. La distribución desigual entre hombres y mujeres interpela el alcance hacia la igualdad en los distintos ámbitos de la sociedad para las mujeres.

Aún el debate actual sobre el valor del trabajo doméstico y del cuidado sigue vigente, así como las implicancias que tiene para los Estados en la creación de una mayor oferta de servicios públicos, sobre todo por el aumento de las demandas futuras que se requerirán para una población que envejece y extiende su esperanza de vida. En estas circunstancias, se generan tensiones para muchas mujeres que aún siguen siendo las principales responsables del trabajo doméstico y del cuidado; y es que, además, de las responsabilidades asumidas en el trabajo remunerado, el trabajo doméstico sigue siendo parte de los conflictos de género.

Por otro lado, la preocupación por la precarización de los empleos y las consecuencias de la desprotección social plantean serias implicancias para las políticas públicas, las familias y las empresas. 


\section{Referencias bibliográficas}

Aguirre, R. y Ferrari, F. 2017. Las encuestas sobre el uso del tiempo y trabajo no remunerado en América Latina y el Caribe. Caminos recorridos y desafios hacia el futuro (pp. 14-29). Serie Asuntos de Género. Santiago de Chile: CEPAL. https://cutt. ly/dUnWHZY

Borderías, C., Carrasco, C. y Alemany, C. 1994. Las mujeres y el trabajo. Rupturas conceptuales. Barcelona: Icaria.

Comisión Económica para América Latina y el Caribe (CEPAL). 2021. Panorama social de América Latina 2020. Santiago de Chile: CEPAL.

Comisión Económica para América Latina y el Caribe (CEPAL). 2019. Las mediciones de uso del tiempo en América Latina y el Caribe. Sistemas de información: transformar datos en información, información en conocimiento y conocimiento en decisión politica. Santiago de Chile: CEPAL. https://cutt.ly/pUnWBtv

Comisión Económica para América Latina y el Caribe (CEPAL). 2014. Desigualdades de género en el mercado laboral y sus efectos sobre la desigualdad socioeconómica: ¿cuánto se podría avanzar? En Panorama social de América Latina, 2014 (capítulo IV). Santiago de Chile: CEPAL. https://cutt.ly/6UnW4sQ

Comisión Económica para América Latina y el Caribe (CEPAL). 2015. Informe de la 8va. Reunión de la Conferencia de Estadística de las América de la Comisión Económica para América Latina y el Caribe. Quito del 17 al 19 de noviembre de 2015. https:// cutt.ly/2UnEri4

Departamento Administrativo Nacional de Estadística (DANE). 2021. Boletín Técnico de la Encuesta de Uso del Tiempo (ENUT). https://cutt.ly/vUnEdmk

Departamento Administrativo Nacional de Estadística (DANE). 2018. Boletín Técnico 2017. Cuenta Satélite de Economía del Cuidado-CSEC. Bogotá: DANE. https://cutt. ly/KUnEWVP

Guzmán, V. y Mauro, A. (2004). Las trayectorias laborales de mujeres de tres generaciones: coacción y autonomía. En El trabajo se transforma. Relaciones de producción y relaciones de género (pp. 207-246). Santiago de Chile: Centro de Estudios de la Mujer. https://cutt.ly/vUnEIOW

Instituto Nacional de Estadística y Censos (INEC). 2020. Boletín Técnico N. ${ }^{\circ}$ 01-2020 CSTNRH. Cuenta Satélite del Trabajo no Remunerado de los Hogares 2016-2017. Quito: INEC. https://cutt.ly/vUnEFZG

Instituto Nacional de Estadística y Censos (INEC). 2019. Publicación de los resultados de las Cuentas Satélites del Trabajo Doméstico no Remunerado de los Hogares 2007-2015. Quito: INEC. 
Instituto Nacional de Estadística e Informática. (INEI). 2019. Empleo y Previsión Social. En Compendio estadístico Perú 2019 (capítulo 7). Lima: INEI. https://cutt.ly/ jUnRDxd

Instituto Nacional de Estadística e Informática. (INEI). 2018. Perú: Indicadores de empleo e ingresos por departamento 2007-2017. Lima: INEI. https://cutt.ly/OUnRKwE Instituto Nacional de Estadística e Informática. (INEI). 2016. Cuenta Satélite del Trabajo Doméstico no Remunerado. Lima: INEI. https://cutt.ly/3UnRXDg

Instituto Nacional de Estadística e Informática. (INEI). 2011. Encuesta Nacional de Uso del Tiempo 2010. Principales resultados. Lima: INEI. https://cutt.ly/0UnRMzd

International Labour Organization (ILOSTAT). 2021. Statistics and databases. https:// cutt.ly/bUnR2KF

International Labour Organization (ILOSTAT). 2021. Statistics on the working-age population and labour force. https://cutt.ly/wUnR7ye

Jelin, E. y Feijoó, M. (1980). Trabajo y familia en el ciclo de vida femenino. El caso de los sectores populares de Buenos Aires. En Las tramas del tiempo: familia, género, memorias, derechos y movimientos sociales. Antología esencial (pp. 77-119). Buenos Aires, CLACSO. https://cutt.ly/uUnTqHm

Organización Internacional del Trabajo (OIT) 1982. Resolución sobre estadísticas de la población económicamente activa, del empleo, del desempleo y del subempleo. Décimo Tercera Conferencia Internacional de Estadígrafos del Trabajo. https://cutt. ly/gUnTreM

Organización Internacional del Trabajo (OIT). 2009. La igualdad de género como eje del trabajo decente. Conferencia Internacional del Trabajo, 98. a . Informe VI. https://cutt. ly/EUnTuU6

Picchio, A. 1994. El trabajo de reproducción, tema central en el análisis del mercado laboral. En Borderías, C., Carrasco, C. y Alemany, C. (Eds.). Las mujeres y el trabajo. Rupturas conceptuales. Barcelona: Icaria. 